\title{
SPECTRAL CURVES AND WHITHAM EQUATIONS IN ISOMONODROMIC PROBLEMS OF SCHLESINGER TYPE*
}

\author{
KANEHISA TAKASAKI ${ }^{\dagger}$
}

\begin{abstract}
It has been known since the beginning of this century that isomonodromic problems - typically the Painlevé transcendents - in a suitable asymptotic region look like a kind of "modulation" of isospectral problem. This connection between isomonodromic and isospectral problems is reconsidered here in the light of recent studies related to the Seiberg-Witten solutions of $N=2$ supersymmetric gauge theories. A general machinary is illustrated in a typical isomonodromic problem, namely the Schlesinger equation, which is reformulated to include a small parameter $\epsilon$. In the small- $\epsilon$ limit, solutions of this isomonodromic problem are expected to behave as a slowly modulated finite-gap solution of an isospectral problem. The modulation is caused by slow deformations of the spectral curve of the finite-gap solution. A modulation equation of this slow dynamics is derived by a heuristic method. An inverse period map of Seiberg-Witten type turns out to give general solutions of this modulation equation. This construction of general solution also reveals the existence of deformations of Seiberg-Witten type on the same moduli space of spectral curves. A prepotential is also constructed in the same way as the prepotential of the Seiberg-Witten theory.
\end{abstract}

1. Introduction. The notion of "isomonodromic deformations" was first discovered by R. Fuchs [1] in 1907 as a new interpretation of the 6th Painlevé equation $\left(\mathrm{P}_{\mathrm{VI}}\right)$, and developed in diverse directions in the next decade. R. Fuchs considered a second order scalar ODE

$$
\frac{d^{2} y}{d \lambda^{2}}+p(\lambda) y=0
$$

of Fuchsian type with four regular singularities, one of which is an apparent singularity. Garnier [2] generalized the work of R. Fuchs in two different forms. One generalization is to consider more than four regular singularities. This leasd to a multi-variable generalization of $\mathrm{P}_{\mathrm{VI}}$. The other is to include irregular singularities. The other five Painlevé equations $\left(P_{I}-P_{V}\right)$ can be derived from this generalization. Schlesinger [3] obtained the so called Schlesinger equation from isomonodromic deformations of first order matrix ODE

$$
\frac{d Y}{d \lambda}=M(\lambda) Y
$$

where $M(\lambda)$ is an $r \times r$ matrix of the form

$$
M(\lambda)=\sum_{i=1}^{N} \frac{A_{i}}{\lambda-t_{i}} .
$$

Garnier noticed later [4] that the $2 \times 2$ Schlesinger equation is equivalent to his isomonodromic deformations of 2 nd order scalar Fuchsian equation.

It is also in this decade that a link with "isospectral deformations" was uncovered. This is again due to Garnier [5]. He proposed an autonomous analogue of the Schlesinger equations, and pointed out that it can be integrated by Abelian functions.

*Received July 16, 1998; accepted for publication November 30, 1998.

$\dagger$ Department of Fundamental Sciences, Kyoto University Yoshida, Sakyo-ku, Kyoto 606, Japan (takasaki@yukawa.kyoto-u.ac.jp). Research partly supported by the Grant-in-Aid for Scientific Researches, Priority Area 231 "Infinite Analysis", the Ministry of Education, Science and Culture, Japan. 
Remarkably, Garnier substantially arrived at the notion of isospectral deformations therein. Let us briefly review Garnier's discovery. Schlesinger's equation can be written

$$
\frac{\partial A_{i}}{\partial t_{j}}=\left(1-\delta_{i j}\right) \frac{\left[A_{i}, A_{j}\right]}{t_{i}-t_{j}}-\delta_{i j} \sum_{k(\neq i)} \frac{\left[A_{i}, A_{k}\right]}{t_{i}-t_{k}}
$$

This is a non-autonomous system, because the right hand side contains the independent variables $t=\left\{t_{i}\right\}$ explicitly. Garnier's proposal was to replace these $t_{i}$ 's by constants, $t_{i} \rightarrow c_{i}$. The outcome is an autonomous system of the form

$$
\frac{\partial A_{i}}{\partial t_{j}}=\left(1-\delta_{i j}\right) \frac{\left[A_{i}, A_{j}\right]}{c_{i}-c_{j}}-\delta_{i j} \sum_{k(\neq i)} \frac{\left[A_{i}, A_{k}\right]}{c_{i}-c_{k}} .
$$

As Garnier noticed, this gives isospectral deformations of $M(\lambda)$, namely, the characteristic polynomial $\operatorname{det}(M(\lambda)-\mu I)$ is invariant under the $t$ flows. The algebraic curve defined by the characteristic equation

$$
\operatorname{det}(M(\lambda)-\mu I)=0
$$

in the $(\lambda, \mu)$ plane (and its appropriate compactification) is nowadays called the "spectral curve." What Garnier did is to solve the above autonomous system in terms of Abelian functions on this algebraic curve.

Isospectral problems of the same type were studied by Moser [6] in the 70's. Moser proposed a matrix nonlinear system as a unified framework for a number of classically well known completely integrable dynamicals systems, such as the Neumann and Rosochatius systems, geodesic flows on an ellipsoid, etc. Moser's idea and Mumford's related work [7] were reformulated by the Montreal group (Adams, Harnad, Hurtubise and Previato) $[8,9,10]$ and Beauville $[11]$ to the isospectral problem of a rational matrix of the form $M(\lambda)$ (or $U+M(\lambda)$, where $U$ is a constant matrix). Harnad and his collaborators later applied their method to isomonodromic problems $[12,13,14,15]$.

Garnier's proposal, however, originally aimed at a quite different issue. He considered the autonomous system as a tool for studying asymptotic behavior of solutions of the Schlesinger equation in a neighborhood of singularities. A similar problem concerning the Painlevé equations had been pursued by Boutroux [16]. Boutroux obtained an asymptotic expression of Painlevé transcendents as a "modulated" elliptic function. Here "modulation" means that parameters of the elliptic function also depend (but "slowly") on the independent variable. In Garnier's program, Boutroux's elliptic curve is replaced by a more general algebraic curve. Flaschka and Newell $[17,18]$ revisited Garnier's program in their study of isomonodromic and isospectral problems, and noted an important remark: They pointed out that a JWKB approximation converts the monodromy problem into a spectral problem.

We reconsider this issue in the light of researches in the last ten years. Since the end of the 80 's, low dimensional string and topological field theories have provided new subjects of isomonodromic problems. A central subject is the so called "string quations" of two-dimensional quantum gravity [19]. They are the first (or second) Painlevé equation and its higher dimensional generalizations. These string equations have been studied from several different points of view. Among them, we are particularly interested in the approach [20, 21, 22, 23] from the "Whitham averaging method" [24] (also referred to as the "nonlinear JWKB method" [25]). This method 
may be viewed as a modernized version of Boutroux's analysis. We now consider the Schlesinger equation in the same philosophy.

The Whitham averaging method, or the nonlinear JWKB method, covers a wide area of modulational phenomena in nonlinear waves. The most relevant for our problem is the case of modulation of Abelian function solutions (which are usually called "finite-gap" or "quasi-periodic" solutions $[26,27,28])$ to a soliton equation. An "unmodulated" finite-band solution generally takes the form

$$
u_{0}=u_{0}\left(\sum_{i} U_{i} t_{i} \mid\left\{I_{n}\right\}\right)
$$

where $u_{0}$ is an Abelian function, $U_{i}$ 's are $g$-dimensional constant vectors ( $g$ is the genus of the spectral curve), and $I_{n}$ 's are other parameters of the solution. The parameters $U_{i}$ 's and $I_{n}$ 's are eventually determined by the spectral curve (as period integrals of meromorphic differentials). If the problem in question contains a small parameter $\epsilon$, one may consider a solution with the following asymptotic form

$$
u \sim u_{0}\left(\sum_{i} U_{i}(T) t_{i} \mid\left\{I_{n}\right\}\right)
$$

as $\epsilon \rightarrow \infty$. The parameter $U_{i}$ and $I_{n}$ now depend on the "slow variables"

$$
T=\left\{T_{i}\right\}, \quad T_{i}=\epsilon t_{i} .
$$

This is the "modulation" of a finite-gap solution. The Whitham averaging method is a method to determine this slow dynamics in $T_{i}$ in the form of differential equations. This kind of differential equations are generally called "modulation equations" (or "Whitham equations"). In the case of finite-gap solutions, the modulation equation can be formulated as a dynamical system on the moduli space of spectral curves.

These modulation equations of finite-gap solutions are known to possess a number of remarkable properties [29]. It is Flaschka, Forest and McLaughlin [30] who first pointed out that this type of modulation equations have a universal structure. They demonstrated, in the case of the $\mathrm{KdV}$ equation, that the modulation equation boils down to the universal form

$$
\frac{\partial}{\partial T_{i}} d \Omega_{j}=\frac{\partial}{\partial T_{j}} d \Omega_{i}
$$

where $d \Omega_{i}$ 's are meromorphic differentials on the spectral curve. Krichever [31, 32] and Dubrovin [33,34] presented an abstract reformulation of this type of equations ("Whitham hierarchies"), and constructed many special solutions (with applications to geometry and physics).

Our concern lies in special solutions of the universal Whitham hierarchy that represent slow dynamics of a spectral curve in isomonodromic problems. Remarkably, it seems likely that this class of solutions of the universal Whitham hierarchy are always characterized by a differential equation of the form

$$
\frac{\partial}{\partial T_{i}} d S=d \Omega_{i}, \quad d S=\mu d \lambda
$$

This is indeed the case for the string equations [20, 21, 22, 23]. In this paper, we shall derive a modulation equation of this form from the Schlesinger equation. 
We use a very heuristic method to derive the modulation equation. This heuristic method was developed in an attempt [35] at an isomonodromic interpretation of integrable structures in supersymmetric gauge theories [36]-[47]. The modulation equation turns out to possess almost the same properties as the so called "Seiberg-Witten solutions" of $N=2$ supersymmetric gauge theories. In particular, we introduce a period map of the Seiberg-Witten type, and prove that the inverse period map solves the modulation equation. This also reveals the existence of another set of commuting flows on the moduli space of spectral curves. We also show that the notion of prepotential can be generalized to this case. These results will be strong evidence for the validity of the heuristic derivation.

This paper is organized as follows. Sections 2 and 3 are of preliminary nature. In Section 2, we review basic properties of the Schlesinger equation. In Section 3, we consider the geometric structure of spectral curves along the lines of approach by the Montreal group and Beauville. Section 4, 5, and 6 are focussed on the derivation of the modulation equation. We begin with a reformulation of the Schlesinger equation in Section 4. The reformulated Schlesinger equation has a small parameter $\epsilon$. Garnier's autonomous system emerges in the limit of $\epsilon \rightarrow 0$. Our modulation equation is derived in Section 5, along with comments on other possible approaches. The structure of meromorphic differentials $d \Omega_{i}$, which are also basic constituents of our modulation equation, is specified in Section 6. Section 7 is devoted to solving the modulation equation by the inverse period map. Section 8 deals with the notion of prepotential. We conclude this paper in Section 9.

2. Schlesinger equation. In this section, we review basic properties of the Schlesinger equation. For details and related topics, we refer to a series of papers by the Kyoto school (Jimbo, Miwa, Môri, Sato and Ueno) [48, 49, 50, 51].

2.1. Coadjoint orbit and hamiltonian structure. Let $\operatorname{gl}(r, \mathbf{C})^{N}$ denote a direct sum of $N$ copies of $\operatorname{gl}(r, \mathbf{C})$. This is the space of $N$-tuples $\left(A_{1}, \cdots, A_{N}\right)$ of $r \times r$ matrices. GL $(r, \mathbf{C})$ acts on this space by the diagonal coadjoint action: $A_{i} \mapsto g A_{i} g^{-1}$. Since the Schlesinger equation can be written

$$
\frac{\partial A_{i}}{\partial t_{j}}=\left[A_{i},\left(1-\delta_{i j}\right) \frac{A_{j}}{t_{i}-t_{j}}-\delta_{i j} \sum_{k(\neq i)} \frac{A_{k}}{t_{i}-t_{k}}\right]
$$

each coadjoint orbit $\mathcal{O}_{i}$ in the $i$-th component of $\operatorname{gl}(r, \mathbf{C})^{N}$ is left invariant under the $t$-flows. Thus the Schlesinger equation is actually a collection of non-autonomous dynamical systems on a direct product $\mathcal{O}_{1} \times \cdots \times \mathcal{O}_{N}$ of coadjoint orbits in $\operatorname{gl}(r, \mathrm{C})$. Usually, only semi-simple orbits are considered; such an orbit is labeled by the eigenvalues $\theta_{i \alpha}(\alpha=1, \cdots, r)$ of $A_{i}$. In other words, these eigenvalues (and, in general, the Jordan canonical form of $A_{i}$ 's) are invariants of the Schlesinger equation.

Actually, there are some extra invariants. They are the matrix elements of

$$
A_{\infty}=-\sum_{i=1}^{N} A_{i}
$$

which are invariant under the Schlesinger equation:

$$
\frac{\partial A_{\infty}}{\partial t_{i}}=0
$$


This matrix, too, is usually assumed to be semi-simple, and it is customary to diagonalize this matrix in advance by a constant "gauge transformation" $A_{i} \mapsto C A_{i} C^{-1}$. Thus only the eigenvalues $\theta_{\infty \alpha}(\alpha=1, \cdots, r)$ of $A_{\infty}$ are nontrivial invariants.

Geometrically, this gauge-fixing may be interpreted as the Marsden-Weinstein construction of a "reduced phase space." The "unreduced phase space" is a coadjoint orbit $\mathcal{O}_{1} \times \cdots \mathcal{O}_{N} \times \mathcal{O}_{\infty}$ in the vector space $\operatorname{gl}(r, \mathbf{C})^{N+1}$ of $(N+1)$-tuples $\left(A_{1}, \cdots, A_{N}, A_{\infty}\right)$. In order to reproduce the Schlesinger equation, one has to impose the linear constraint

$$
\sum_{i=1}^{N} A_{i}+A_{\infty}=0
$$

and "gauge away" redundant degrees of freedom by the action of GL $(r, \mathbf{C})$. The left hand side of the linear constraint is essentially a moment map of this diagonal $\mathrm{GL}(r, \mathbf{C})$ action.

Analytically, as we see show below, the coadjoint orbit invariants $\theta_{i \alpha}(i=1,2, \cdots$, $N, \infty, \alpha=1, \cdots, r)$ give local monodromy exponents of Schlesinger's monodromy problem.

The coadjoint orbit structure leads to a Hamiltonian formalism of the Schlesinger equation. Let us introduce a Poisson structure on the vector space $\operatorname{gl}(r, \mathbf{C})^{N}$ by defining the Poisson bracket of matrix elements of $A_{i}=\left(A_{i, \alpha \beta}\right)$ as:

$$
\left\{A_{i, \alpha \beta}, A_{j, \rho \sigma}\right\}=\delta_{i j}\left(-\delta_{\beta \rho} A_{i, \alpha \sigma}+\delta_{\sigma \alpha} A_{i, \rho \beta}\right) .
$$

In each component of the direct sum, this is just the ordinary Kostant-Kirillov Poisson bracket. The Schlesinger equation can be written in the Hamiltonian form

$$
\frac{\partial A_{j}}{\partial t_{i}}=\left\{A_{j}, H_{i}\right\}
$$

where the Hamiltonians are given by

$$
H_{i}=\operatorname{Res}_{\lambda=T_{i}} \frac{1}{2} \operatorname{Tr} M(\lambda)^{2}=\sum_{j(\neq i)} \operatorname{Tr}\left(\frac{A_{i} A_{j}}{t_{i}-t_{j}}\right),
$$

and involutive,

$$
\left\{H_{i}, H_{j}\right\}=0 \text {. }
$$

2.2. Isomonodromic deformations. The Schlesinger equation gives isomonodromic deformations of the first order ODE

$$
\frac{d Y}{d \lambda}=M(\lambda) Y
$$

with the rational coefficient matrix

$$
M(\lambda)=\sum_{i=1}^{N} \frac{A_{i}}{\lambda-t_{i}}
$$

Note that $A_{\infty}$ is the residue of $M(\lambda)$ at $\lambda=\infty$.

Usually, this type of isomonodromic problems are considered under the following 


\section{AsSumption}

- The residue matrices $A_{i}(i=1, \cdots, N, \infty)$ are diagonalizable.

- The eigenvalues $\theta_{i \alpha}(\alpha=1, \cdots, r)$ of each residue matrix $A_{i}$ have no integer difference, i.e., $\theta_{i \alpha}-\theta_{i \beta} \notin \mathbf{Z}$ if $\alpha \neq \beta$.

We assume them throughout this paper. These assumptions ensure that local solutions at the singular points $\lambda=t_{1}, \cdots, t_{N}, \infty$ develop no logarithmic term (see below).

The isomonodromic deformations are generated by the deformation equations

$$
\frac{\partial Y}{\partial t_{i}}=-\frac{A_{i}}{\lambda-t_{i}} Y
$$

These deformation equations and the above first order ODE comprise an "auxiliary linear problem" of the Schlesinger equation. Its Frobenius integrability conditions can be written in the "zero-curvature form"

$$
\left[\frac{\partial}{\partial t_{j}}+\frac{A_{i}}{\lambda-t_{i}}, M(\lambda)-\frac{\partial}{\partial \lambda}\right]=0, \quad\left[\frac{\partial}{\partial t_{i}}+\frac{A_{i}}{\lambda-t_{i}}, \frac{\partial}{\partial t_{j}}+\frac{A_{j}}{\lambda-t_{j}}\right]=0
$$

and one can easily check that these zero-curvature equations are equivalent to the Schlesinger equation.

2.3. Local solutions at singular points and tau function. Since $\lambda=$ $t_{1}, \cdots, t_{N}$ and $\lambda=\infty$ are regular singularities of the above first order ODE, one can construct a local solution of the following form at each of these singular points:

- Local solution at $\lambda=t_{i}$ :

$$
Y_{i}=\hat{Y}_{i} \cdot\left(\lambda-t_{i}\right)^{\Theta_{i}}, \quad \hat{Y}_{i}=\sum_{n=0}^{\infty} Y_{i n}\left(\lambda-t_{i}\right)^{n} .
$$

- Local solution at $\lambda=\infty$ :

$$
Y_{\infty}=\hat{Y}_{\infty} \cdot \lambda^{-\Theta_{\infty}}, \quad \hat{Y}_{\infty}=\sum_{n=0}^{\infty} Y_{\infty n} \lambda^{-n}
$$

Here $Y_{i n}$ are $r \times r$ matrices, the leading coefficients $Y_{i 0}$ and $Y_{\infty 0}$ are invertible, and $\Theta_{i}$ and $\Theta_{\infty}$ are diagonal matrices of local monodromy exponents. Inserting these expressions into the first order ODE gives the relations

$$
A_{i}=Y_{i 0} \Theta_{i} Y_{i 0}^{-1}, \quad i=1, \cdots, N, \infty
$$

In particular, local monodromy exponents coincide with the eigenvalues of $A_{i}$ :

$$
\Theta_{i}=\operatorname{diag}\left(\theta_{i 1}, \cdots, \theta_{i r}\right) .
$$

It is not hard to check that these expressions of $A_{i}$ and $\Theta_{i}$ are also consistent with the other equations of the auxiliary linear problem.

The $\tau$ function of the Schlesinger equation is defined in two equivalent ways. One way is to define $\log \tau$ as a potential of the Hamiltonians $H_{i}$ :

$$
d \log \tau=\sum_{i=1}^{N} H_{i} d t_{i}
$$


Another equivalent definition, which is more suited for generalization, is based on the equations

$$
\frac{\partial \log \tau}{\partial t_{i}}=\operatorname{Tr} \Theta_{i} Y_{i 0}^{-1} Y_{i 1}
$$

The equivalence can be verified as follows:

$$
\begin{aligned}
H_{i} & =\sum_{j(\neq i)} \operatorname{Tr} \frac{A_{i} A_{j}}{t_{i}-t_{j}} \\
& =\operatorname{Res}_{\lambda=t_{i}} \operatorname{Tr} \frac{A_{i}}{\lambda-t_{i}} M(\lambda) \\
& =\underset{\lambda=t_{i}}{\operatorname{Res}} \operatorname{Tr} \frac{Y_{i 0} \Theta_{i} Y_{i 0}^{-1}}{\lambda-t_{i}} \frac{\partial Y_{i}}{\partial \lambda} Y_{i}^{-1} \\
& =\underset{\lambda=t_{i}}{\operatorname{Res}} \operatorname{Tr} \frac{\Theta_{i}}{\lambda-t_{i}} Y_{i 0}^{-1} \frac{\partial Y_{i}}{\partial \lambda} Y_{i}^{-1} Y_{i 0} \\
& =\operatorname{Tr} \Theta_{i} Y_{i 0}^{-1} Y_{i 1} .
\end{aligned}
$$

The closedness of the 1 -form $\sum H_{i} d t_{i}$, or equivalently the integrability condition

$$
\frac{\partial H_{i}}{\partial t_{j}}=\frac{\partial H_{j}}{\partial t_{i}}
$$

is ensured by the Schlesinger equation itself.

3. Spectral curve. By "spectral curve," we mean the plane algebraic curve defined on the $(\lambda, \mu)$ plane by

$$
\operatorname{det}(M(\lambda)-\mu I)=0
$$

and its suitable compactification. We first discuss its roles in isomonodromic and isospectral problems, then consider its geometric properties.

3.1. Spectral curve in isomonodromic problem. Isomonodromic deformations such as the Schlesinger equations are non-isospectral, namely, the spectral curve varies in deformations.

Let us present an interesting formula (essentially due to Vereschagin [52]) which show qualitatively that the characteristic polynomial of $M(\lambda)$ varies under isomonodromic deformations. First, by the the well known identities of linear algebra, we have

$$
\begin{aligned}
\frac{\partial}{\partial t_{i}} \log \operatorname{det}(M(\lambda)-\mu I) & =\frac{\partial}{\partial t_{i}} \operatorname{Tr} \log (M(\lambda)-\mu I) \\
& =\operatorname{Tr} \frac{\partial M(\lambda)}{\partial t_{i}}(M(\lambda)-\mu I)^{-1}
\end{aligned}
$$

By the first equation of the zero-curvature representation, $\partial M(\lambda) / \partial t_{i}$ can be rewritten

$$
\frac{\partial M(\lambda)}{\partial t_{i}}=\frac{A_{i}}{\left(\lambda-t_{i}\right)^{2}}-\left[\frac{A_{i}}{\lambda-t_{i}}, M(\lambda)-\mu I\right] .
$$

The second term on the right hand side has no contribution, because of the identity

$$
\operatorname{Tr}\left[\frac{A_{i}}{\lambda-t_{i}}, M(\lambda)-\mu I\right](M(\lambda)-\mu)^{-1}=0 .
$$


Thus, eventually, we obtain the formula

$$
\frac{\partial}{\partial t_{i}} \log \operatorname{det}(M(\lambda)-\mu I)=\operatorname{Tr} \frac{A_{i}}{\left(\lambda-t_{i}\right)^{2}}(M(\lambda)-\mu I)^{-1} .
$$

This clearly shows that the $t$-dependence of the characteristic polynomial of $M(\lambda)$ is driven by the "anomalous" term $A_{i} /\left(\lambda-t_{i}\right)^{2}$.

3.2. Spectral curve in isospectral problem. We now turn to Garnier's autonomous analogue of the Schlesinger equation. This equation has the following zerocurvature representation:

$$
\left[\frac{\partial}{\partial t_{i}}+\frac{A_{i}}{\lambda-c_{i}}, M(\lambda)\right]=0, \quad\left[\frac{\partial}{\partial t_{i}}+\frac{A_{i}}{\lambda-c_{i}}, \frac{\partial}{\partial t_{j}}+\frac{A_{j}}{\lambda-c_{j}}\right]=0 .
$$

Repeating the same calculations as above, we now find that

$$
\frac{\partial}{\partial t_{i}} \operatorname{det}(M(\lambda)-\mu I)=0
$$

because there is no "anomalous" term like $A_{i} /\left(\lambda-t_{i}\right)^{2}$. Thus one can confirm that Garnier's autonomous system is indeed an isospectral problem. An auxiliary linear problem is given by

$$
\mu \psi=M(\lambda) \psi, \quad \frac{\partial \psi}{\partial t_{i}}=-\frac{A_{i}}{\lambda-c_{i}} \psi
$$

Here $\psi$ is understood to be a column vector. The first equation means that $\psi$ is an eigenvector of $M(\lambda)$ with eigenvalue $\mu$. The second set of equations generate isospectral deformations.

It is nowadays well known that this type of isospectral problems can be mapped to linear flows on the Jacobian variety of the spectral curve [26, 27, 28]. Reproducing the original nonlinear problem is identical to Jacobi's inversion problem, and indeed solvable by theta functions (or Baker-Akhiezer functions). The solution thus obtained is written in terms of period integrals and Abelian functions.

Although isomonodromic problems (in a generic case) cannot be solved in that way, the notion of spectral curve still plays a role in the study of Hamiltonian structures. Indeed, Harnad and Wisse $[12,15]$ presented a construction of special Darboux coordinates ("spectral Darboux coordinates") using the language of spectral curves. The case of $r=2$ is particularly interesting, because this is the case where the 6th Painlevé equation $\left(\mathrm{P}_{\mathrm{VI}}\right)$ and Garnier's multi-variable version of $\mathrm{P}_{\mathrm{VI}}$ (called the "Garnier system" by Okamoto [53]) emerge. The spectral Darboux coordinates in this case coincide with Okamoto's Darboux coordinates for the "Garnier system," which Okamoto discovered without using the notion of spectral curve.

3.3. Geometry of spectral curves. The structure of spectral curves of the above type has been elucidated in detail by the Montreal group $[8,9,10]$ and Beauville [11]. We present basic part of their results, which will be used in the subsequent sections. In the following, the poles of $M(\lambda)$ are written $c_{i}$ rather than $T_{i}$. plane:

3.3.1. Spectral curve on plane. Let $C_{0}$ be the spectral curve on the $(\lambda, \mu)$

$$
F(\lambda, \mu)=\operatorname{det}(M(\lambda)-\mu I)=0
$$


This becomes a ramified covering of the punctured Riemann sphere,

$$
\pi: C_{0} \rightarrow \mathbf{C} P^{1} \backslash\left\{c_{1}, \cdots, c_{N}, \infty\right\}, \quad \pi(\lambda, \mu)=\lambda .
$$

For a generic value of $\lambda$, the inverse image $\pi^{-1}(\lambda)$ consists of $r$ points $\left(\lambda, \mu_{\alpha}\right)(\alpha=$ $1, \cdots, r)$. The $\mu$-coordinates $\mu_{\alpha}$ of these points are eigenvalues of $M(\lambda)$. In a neighborhood of $\lambda=c_{i}, \mu_{\alpha}$ 's behave as

$$
\mu_{\alpha}=\frac{\theta_{i \alpha}}{\lambda-c_{i}}+\text { non-singular, }
$$

where $\theta_{i \alpha}$ are the eigenvalues of $A_{i}$. Similarly, in a neighborhood of $\lambda=\infty$,

$$
\mu_{\alpha}=-\theta_{\infty \alpha} \lambda^{-1}+O\left(\lambda^{-2}\right)
$$

where $\theta_{\infty \alpha}$ are the eigenvalues of $A_{\infty}=-\sum_{i=1}^{N} A_{i}$. (Of course, the numbering of $\mu_{\alpha}$ 's is meaningful only locally.)

3.3.2. Compactification of spectral curve. We now compactify $C_{0}$ by adding several points over the punctures of the Riemann sphere. In a neighborhood of $\lambda=c_{i}$, let us consider the following $\tilde{\mu}$ in place of $\mu$ :

$$
\tilde{\mu}=f(\lambda) \mu, \quad f(\lambda)=\prod_{i=1}^{N}\left(\lambda-c_{i}\right) .
$$

In terms of the new coordinates $(\lambda, \tilde{\mu})$, the equation of the spectral curve becomes

$$
\tilde{F}(\lambda, \tilde{\mu})=\operatorname{det}(f(\lambda) M(\lambda)-\tilde{\mu} I)=0 .
$$

The inverse image $\pi^{-1}(\lambda)$ consists of $r$ points $\left(\lambda, \tilde{\mu}_{\alpha}\right)(r=1, \cdots, r)$ such that

$$
\tilde{\mu}_{\alpha}=f^{\prime}\left(c_{i}\right) \theta_{i \alpha}+O\left(\lambda-c_{i}\right)
$$

as $\lambda \rightarrow c_{i}$. Since the eigenvalues $\theta_{i \alpha}(\alpha=1, \cdots, r)$ are pairwise distinct (recall the assumptions in Section 2), we add to $C_{0}$ extra $r$ points $(\lambda, \tilde{\mu})=\left(c_{i}, f^{\prime}\left(c_{i}\right) \theta_{i \alpha}\right)$ to fill the holes above $\lambda=c_{i}$.

Similarly, in a neighborhood of $\lambda=\infty$, we use

$$
\tilde{\mu}=\lambda \mu
$$

in place of $\mu . \pi^{-1}(\lambda)$ now consists of the $r$ points $\left(\lambda, \tilde{\mu}_{\alpha}\right)(r=1, \ldots, r)$ such that

$$
\tilde{\mu}_{\alpha}=-\theta_{\infty \alpha}+O\left(\lambda^{-1}\right)
$$

as $\lambda \rightarrow \infty$. The eigenvalues $\theta_{\infty \alpha}(r=1, \cdots, r)$, too, are pairwise distinct. Therefore we add to $C_{0}$ the $r$ points $(\lambda, \tilde{\mu})=\left(\infty,-\theta_{\infty \alpha}\right)$ to fill the holes above $\lambda=\infty$.

Thus, by adding altogether $r N+r$ points to $C_{0}$, we obtain a compactification $C$ of $C_{0}$. The covering map $\pi$ uniquely extends to $C$, and gives a ramified covering $\pi: C \rightarrow \mathbf{C} P^{1}$ of the Riemann sphere.

3.3.3. Genus of compactified spectral curve. If there are multiple eigenvalues of $A_{i}$, the compactified spectral curve $C$ has singular points (nodes) over $\lambda=c_{i}$. In that case, one has to take a desingularization $\rho: \tilde{C} \rightarrow C$ for further consideration. Fortunately, this does not occur in our case because of the assumptions introduced in Section 2. One can show, by a standard method, that $C$ has the genus

$$
g=\frac{1}{2}(r-1)(r N-r-2) \text {. }
$$


3.3.4. Structure of characteristic polynomial. In order to examine the structure of the characteristic polynomial of $M(\lambda)$ (or, rather, $f(\lambda) M(\lambda)$ ), we take the rational matrix

$$
M^{0}(\lambda)=\sum_{i=1}^{N} \frac{A_{i}^{0}}{\lambda-c_{i}}, \quad A_{i}^{0}=\operatorname{diag}\left(\theta_{i 1}, \cdots, \theta_{i r}\right),
$$

as a reference point on the coadjoint orbit that $M(\lambda)$ belongs to. Now compare the characteristic polynomials $\tilde{F}(\lambda, \tilde{\mu})$ and $\tilde{F}^{0}(\lambda, \tilde{\mu})$ of $f(\lambda) M(\lambda)$ and $f(\lambda) M^{0}(\lambda)$. Note, first, that $\tilde{F}(\lambda, \tilde{\mu})-\tilde{F}^{0}(\lambda, \mu)$ vanishes at $\lambda=c_{i}$. This will be obvious from the following expression of these polynomials as $\lambda \rightarrow c_{i}$ :

$$
\begin{gathered}
\tilde{F}(\lambda, \tilde{\mu})=\operatorname{det}\left(f^{\prime}\left(c_{i}\right) A_{i}-\tilde{\mu} I\right)+O\left(\lambda-c_{i}\right), \\
\tilde{F}^{0}(\lambda, \tilde{\mu})=\operatorname{det}\left(f^{\prime}\left(c_{i}\right) A_{i}^{0}-\tilde{\mu} I\right)+O\left(\lambda-c_{i}\right) .
\end{gathered}
$$

(The two determinants on the right hand side are equal, because $A_{i}$ and $A_{i}^{0}$ are on the same coadjoint orbit.) Therefore $\tilde{F}(\lambda, \tilde{\mu})-\tilde{F}^{0}(\lambda, \mu)$ is divisible by $f(\lambda)$. Furthermore, these characteristic polynomials have the following expansion in powers of $\tilde{\mu}$ :

$$
\begin{gathered}
\tilde{F}(\lambda, \tilde{\mu})=(-\tilde{\mu})^{r}+\operatorname{Tr} M(\lambda)(-\tilde{\mu})^{r-1}+\cdots, \\
\tilde{F}^{0}(\lambda, \tilde{\mu})=(-\tilde{\mu})^{r}+\operatorname{Tr} M^{0}(\lambda)(-\tilde{\mu})^{r-1}+\cdots .
\end{gathered}
$$

Since the first two terms on the right hand side are equal, respectively, the difference of the two polynomials contains no terms proportional to $\mu^{r}$ and $\mu^{r-1}$. From these observations, one can conclude that the difference of the characteristic functions can be written

$$
\tilde{F}(\lambda, \tilde{\mu})-\tilde{F}(\lambda, \tilde{\mu})=f(\lambda) \sum_{\ell=2}^{r} p_{\ell}(\lambda) \tilde{\mu}^{r-\ell},
$$

where $p_{\ell}(\lambda)$ 's are polynomial functions of $\lambda$. A simple power counting argument (assigning weight 1 to $\lambda$ and weight $N-1$ to $\tilde{\mu}$ ) shows that the degree of $p_{\ell}(\lambda)$ does not exceed the positive integer

$$
\delta_{\ell}=(N-1) \ell-N
$$

Therefore $p_{\ell}(\lambda)$ can be written

$$
p_{\ell}(\lambda)=\sum_{m=0}^{\delta_{\ell}} h_{m \ell} \lambda^{m} .
$$

The leading coefficient of this polynomial is a function of $\theta_{i \alpha}$ 's only, because it can be writen

$$
h_{\delta_{\ell}, \ell}=(-1)^{r}\left(\sigma_{\ell}\left(A_{\infty}\right)-\sigma_{\ell}\left(A_{\infty}^{0}\right)\right) \text {, }
$$

where $\sigma_{\ell}\left(A_{\infty}\right)$ and $\sigma_{\ell}\left(A_{\infty}^{0}\right)$ are the $\ell$-th elementary symmetric function of $A_{\infty}$ and $A_{\infty}^{0}=-\sum_{i=1}^{r} A_{i}^{0}$. Thus, eventually, we arrive at the following expression of $\tilde{F}(\lambda, \tilde{\mu})$ :

$$
\tilde{F}(\lambda, \tilde{\mu})=\tilde{F}^{0}(\lambda, \tilde{\mu})+\sum_{\ell=2}^{r} \sum_{m=0}^{\delta_{\ell}} h_{m \ell} \lambda^{m} \tilde{\mu}^{r-\ell} .
$$


3.3.5. Parameters of spectral curve. Apart from the leading coefficients, the coefficients $h_{m \ell}\left(\ell=2, \cdots, r, m=0, \cdots, \delta_{\ell}-1\right)$ of $p_{\ell}(\lambda)$ 's give arbitrary parameters ("moduli") of the spectral curve. Their total number coincides with the genus of the spectral curve:

$$
\sum_{\ell=2}^{r} \delta_{\ell}=\sum_{\ell=2}^{r}((N-1) \ell-N)=g .
$$

In the isospectral problem, these parameters $h_{m \ell}$ are constants of motion (Hamiltonians of commuting isospectral flows). They should not be confused with the Hamiltonians $H_{i}$ of the isomonodromic deformations.

Actually, the characteristic polynomial has yet another set of parameters - the position $c_{i}$ of poles of $M(\lambda)$. They play the role of deformation variables in isomonodromic problem.

In summary, the spectral curve has three distinct sets of parameters:

- Position of poles $c_{i}(i=1, \cdots, N)$.

- Coadjoint orbit invariants $\theta_{i \alpha}(i=1, \cdots, N, \infty)$.

- Isospectral invariants $h_{m \ell}\left(\ell=2, \cdots, r, m=0, \ldots, \delta_{\ell}-1\right)$.

\section{Schlesinger equation with small parameter.}

4.1. Reformulation including small parameter. The first step towards the derivation of our modulation equation is to reformulate the Schlesinger equation by the following substitution rule:

$$
\frac{\partial}{\partial t_{i}} \rightarrow \epsilon \frac{\partial}{\partial T_{i}}, \quad \frac{\partial}{\partial \lambda} \rightarrow \epsilon \frac{\partial}{\partial \lambda}, \quad \frac{A_{i}}{\lambda-t_{i}} \rightarrow \frac{A_{i}}{\lambda-T_{i}} .
$$

Note, in particular, that the deformation variables are renamed as $t_{i} \rightarrow T_{i} . T_{i}$ 's will play the role of "slow variables." The reformulated Schlesinger equation reads:

$$
\epsilon \frac{\partial A_{i}}{\partial T_{j}}=\left(1-\delta_{i j}\right) \frac{\left[A_{i}, A_{j}\right]}{T_{i}-T_{j}}-\delta_{i j} \sum_{k(\neq i)} \frac{\left[A_{i}, A_{k}\right]}{T_{i}-T_{k}} .
$$

An auxiliary linear problem is given by

$$
\epsilon \frac{\partial Y}{\partial \lambda}=M(\lambda) Y, \quad \epsilon \frac{\partial Y}{\partial T_{i}}=-\frac{A_{i}}{\lambda-T_{i}} Y .
$$

The above $\epsilon$-dependent Schlesinger equation can be indeed reproduced from the the Frobenius integrability conditions

$$
\begin{gathered}
{\left[\epsilon \frac{\partial}{\partial T_{j}}+\frac{A_{i}}{\lambda-T_{i}}, M(\lambda)-\epsilon \frac{\partial}{\partial \lambda}\right]=0,} \\
{\left[\epsilon \frac{\partial}{\partial T_{i}}+\frac{A_{i}}{\lambda-T_{i}}, \epsilon \frac{\partial}{\partial T_{j}}+\frac{A_{j}}{\lambda-T_{j}}\right]=0 .}
\end{gathered}
$$

A few comments on the above reformulation will be in order:

(i) Reformulating the Schlesinger equation as above is inspired by the work of Vereschagin [52], who considered all the six Painlevé equations $\left(\mathrm{P}_{\mathrm{I}}-\mathrm{P}_{\mathrm{VI}}\right)$ in such an $\epsilon$-dependent form. This is different from the way Boutroux [16] and Garnier [5] derived an Abelian function approximation, but, as Vereschagin stresses, they are asymptotically related.

(ii) The string equations of two-dimensional quantum gravity, which are $\mathrm{P}_{\mathrm{I}}, \mathrm{P}_{\mathrm{II}}$ and their higher order generalization, contain such a small parameter from the beginning. The small parameter is interpreted as "string coupling constant" [19]. 
4.2. Isospectral deformations as first approximation. One can now see, at least intuitively, that an isospectral problem emerges from the above $\epsilon$-dependent Schlesinger equation. Let us introduce the "fast variables"

$$
t_{i}=\epsilon^{-1} T_{i}
$$

and rewrite the above equation as

$$
\frac{\partial A_{i}}{\partial t_{j}}=\left(1-\delta_{i j}\right) \frac{\left[A_{i}, A_{j}\right]}{T_{i}-T_{j}}-\delta_{i j} \sum_{k(\neq i)} \frac{\left[A_{i}, A_{k}\right]}{T_{i}-T_{k}}
$$

Suppose we now observe this system in the scale of the fast variables $t_{i}$. In this scale, $T_{i}$ 's may be treated as being approximately constant, because a finite displacement in $t_{i}$ 's corresponds to a small (i.e., $O(\epsilon)$ ) displacement in $T_{i}$ 's. If $T_{i}$ 's were true constants, the above equation would be exactly Garnier's autonomous system. Thus, observed in the scale of the fast variables, our $\epsilon$-dependent Schlesinger equation looks approximately like an isospectral problem.

Of course, this is no more than an approximation. In fact, $T_{i}$ 's are not constant, but vary slowly in the order of $O(\epsilon)$. Accordingly, the spectral curve, too, deforms slowly because the defining equation of the spectral curve contains $T_{i}$ 's explicitly. A precise description of the isospectral approximation has to take into account such slow deformations of the spectral curve.

It is, however, not only $T_{i}$ 's that vary; the isospectral invariants $h_{m \ell}$ also change values slowly. They are both responsible for deformations of the spectral curve. In order to see how this occurs, recall the derivative formula of the characteristic polynomial under isomonodromic deformations (Section 3.1). In the present setting, this formula takes the form

$$
\frac{\partial}{\partial T_{i}} \log \operatorname{det}(M(\lambda)-\mu I)=\operatorname{Tr} \frac{A_{i}}{\left(\lambda-T_{i}\right)^{2}}(M(\lambda)-\mu I)^{-1} .
$$

The right hand side plays the role of a "driving force" for slow deformations of the characteristic polynomial. More precisely, in order to extract a true driving force of slow deformations, one has to take an average of the right hand side over the quasi-periodic motion in the fast variables $t_{i}$. This is a central idea of Vereschagin's averaging method $[23,52]$.

Meanwhile, the coadjoint orbit invariants $\theta_{i \alpha}$ remain constant, because they are also invariant of isomonodromic deformations.

4.3. Concept of multiscale analysis. Although frequently lacking mathematical rigor, "multiscale analysis" is widely accepted in applied mathematics as a powerful tool for dealing with this kind of problems $[54,55,56]$ The Whitham averaging method, too, has been developed in the framework of multiscale analysis. The nonlinear JWKB method of Dobrokhotov and Maslov [25] is an attempt at a rigorous reformulation of multiscale analysis. Let us show how our problem may be formulated in the language of multiscale analysis.

A key of multiscale analysis is to treat the fast variables $t=\left\{t_{i}\right\}$ and the slow variables $T=\left\{T_{i}\right\}$ as independent variables. $A_{i}$ 's are thus assumed to be a function of $t$ and $T$ (and the small parameter $\epsilon$ ),

$$
A_{i}=A_{i}(t, T, \epsilon)
$$


The relation $t_{i}=\epsilon^{-1} T_{i}$ is imposed only in the differential equation in question. (For a more precise description, one may introduce a series of "slower" variables $t^{(2)}, t^{(3)}$, $\cdots$, related with $t_{k}$ 's as

$$
t_{i}^{(k)}=\epsilon^{k} t_{i}
$$

For our purpose, only the first two scales are sufficient.)

In this multiscale ansatz, derivative terms in the equation are given by a sum of $t$-derivatives and $T$-derivatives. In terms of differential operators, this amounts to substituting

$$
\epsilon \frac{\partial}{\partial T_{i}} \rightarrow \frac{\partial}{\partial t_{i}}+\epsilon \frac{\partial}{\partial T_{i}}
$$

We now assume an asymptotic expansion of the form

$$
A_{i}(t, T, \epsilon)=A_{i}^{(0)}(t, T)+A_{i}^{(1)}(t, T) \epsilon+\cdots,
$$

and plug all these stuff into the Schlesinger equation. From each order of $\epsilon$, we obtain a differential equation for the coefficients of the above expansion.

The lowest order equation is give by

$$
\frac{\partial A_{i}^{(0)}}{\partial t_{j}}=\left(1-\delta_{i j}\right) \frac{\left[A_{i}^{(0)}, A_{j}^{(0)}\right]}{T_{i}-T_{j}}-\delta_{i j} \sum_{k(\neq i)} \frac{\left[A_{i}^{(0)}, A_{k}^{(0)}\right]}{T_{i}-T_{k}} .
$$

If we consider $T_{i}=c_{i}$, this is nothing but Garnier's autonomous system. Note that this derivation of Garnier's isospectral problem is more understandable than the intuitive derivation in the last subsection. In the derivation of the last subsection, $t$ and $T$ were not independent and constrained by the relation $t_{i}=\epsilon^{-1} T_{i}$, thereby we had to say that $T$ is "approximately constant"; in the setting of multiscale analysis, $t$ and $T$ are independent. This shows a conceptual advantage of multiscale analysis.

The lowest order equation, however, carries no information on the $T$-dependence of $A_{i}^{(0)}$ 's, which are to be determined by the next order equation. The next order equation is given by

$$
\begin{aligned}
\frac{\partial A_{i}^{(1)}}{\partial t_{j}}= & \left(1-\delta_{i j}\right) \frac{\left[A_{i}^{(0)}, A_{j}^{(1)}\right]+\left[A_{i}^{(1)}, A_{j}^{(0)}\right]}{T_{i}-T_{j}} \\
& -\delta_{i j} \sum_{k(\neq i)} \frac{\left[A_{i}^{(0)}, A_{k}^{(1)}\right]+\left[A_{i}^{(1)}, A_{k}^{(0)}\right]}{T_{i}-T_{k}} \\
& +\left(\text { terms containing } A^{(0)} \text { 's and their } T\right. \text {-derivatives only). }
\end{aligned}
$$

This equation contains $T$-derivatives of $A_{i}^{(0)}$ as well as $t$-derivatives of $A_{i}^{(1)}$. A standard prescription of multiscale analysis is to eliminate the latter by "averaging over the $t$ space." This procedure usually takes the form of an "integrability condition" for the above equation to have a solution $A_{i}^{(1)}$ under suitable a boundary condition (e.g., requiring the same quasi-periodicity as $A_{i}^{(0)}$ 's possess).

One thus obtains a differential equation (in $T$ ) for $t$-averaged functionals of $A_{i}^{(0)}$ 's. This is one of various possible expressions ("modulation equations") of modulational dynamics. As Whitham pointed out [24], such a modulation equation frequently appears in the form of "averaged conservation laws." Having this fact in mind, Flaschka, Forest and McLaughlin [30] considered averaged conservation lows of the KdV equation, and derived their compact expression of this problem. 
4.4. Our approach to modulation equation. There are many technical difficulties in deriving a modulation equation from the Schlesinger equation along the line presented above. A main obstacle is the fact that the spectral curve is no longer hyperelliptic for $r>2$. Most attempts in the literature, including the work of Flaschka, Forest and McLaughlin, have been limited to hyperelliptic spectral curves. This considerably reduces the obstacles (though a complete treatment of the problem is still by no means an easy task). The spectral curve of the Schlesinger equation is hyperelliptic only if $r=2$. In the general case, the averaging method is inevitably confronted with delicate problems of geometry of spectral curves. (In this respect, Krichever's averaging method $[57,58]$ for general spectral curves is quite remarkable.)

Since our main concern is the structure of the modulation equation rather than the averaging method itself, we now bypass these delicate issues by a very heuristic argument.

\section{Modulation equation.}

5.1. Multiscale analysis of auxiliary linear problem. The first step of our heuristic argument is to apply the concept of multiscale analysis to the auxiliary linear problem of the Schlesinger equation. Besides the multiscale expression of $A_{i}$ 's, we now assume the following ansatz to $Y$ (which is now understood to be vector-valued):

$$
Y=\left(\phi^{(0)}(t, T, \lambda)+\phi^{(1)}(t, T, \lambda) \epsilon+\cdots\right) \exp \epsilon^{-1} S(T, \lambda) .
$$

Here $\phi^{(k)}(t, T, \lambda)$ are vector-valued function and $S(T, \lambda)$ a scalar-valued function. This is a kind of JWKB ansatz (inspired by the work of Flaschka and Newell $[17,18]$ and Novikov [20]). Note that the "phase function" $S$ is independent of $t$. We can now write the auxiliary linear problem in the following multiscale form:

$$
\begin{array}{r}
\epsilon \frac{\partial Y}{\partial \lambda}=M(\lambda) Y \\
\left(\frac{\partial}{\partial t_{i}}+\epsilon \frac{\partial}{\partial T_{i}}\right) Y=-\frac{A_{i}}{\lambda-T_{i}} Y
\end{array}
$$

The leading order equation should reproduce the auxiliary linear problem of the isospectral problem. Let us confirm that this is indeed the case. The leading order equation is given by

where

$$
\begin{array}{r}
\frac{\partial S(\lambda)}{\partial \lambda} \phi^{(0)}(\lambda)=M^{(0)}(\lambda) \phi^{(0)}(\lambda) \\
\frac{\partial \phi^{(0)}(\lambda)}{\partial t_{i}}+\frac{\partial S(\lambda)}{\partial T_{i}} \phi^{(0)}(\lambda)=-\frac{A_{i}^{(0)}}{\lambda-T_{i}} \phi^{(0)}
\end{array}
$$

We now define

$$
M^{(0)}(\lambda)=\sum_{i=1}^{N} \frac{A_{i}^{(0)}}{\lambda-T_{i}}
$$

$$
\mu=\frac{\partial S(\lambda)}{\partial \lambda}, \quad \psi=\phi^{(0)}(\lambda) \exp \sum_{i=1}^{N} t_{i} \frac{\partial S(\lambda)}{\partial T_{i}}
$$


In terms of these quantities, the leading order equation of multiscale expansion can be rewritten

$$
\mu \psi=M^{(0)}(\lambda) \psi, \quad \frac{\partial \psi}{\partial t_{i}}=-\frac{A_{i}^{(0)}}{\lambda-T_{i}} \psi
$$

This is exactly the auxiliary linear problem of the isospectral problem that we derived in Section 4!

5.2. Matching with Baker-Akhiezer function. The next step is the most crucial part of our heuristics. We now compare the above $\psi$ with the Baker-Akhiezer function in the ordinary algebro-geometric approach $[26,27,28]$ to finite-band solutions of soliton equations.

It is well known that the solution of the auxiliary linear problem for a finite-band solution can be constructed as a (scalar- or vector-valued) Baker-Akhiezer function. In the present setting, such a Baker-Akhiezer function can be written

$$
\psi=\phi \exp \sum_{i=1}^{N} t_{i} \Omega_{i}
$$

Here $\phi$ is a vector-valued function on the spectral curve, also depending on $t$; each entry is a combination of theta functions. We do not specify its detailed structure, because it is irrelevant in the following consideration. Meanwhile, $\Omega_{i}$ is the primitive function of a meromorphic differential $d \Omega_{i}$ on the spectral curve,

$$
\Omega_{i}=\int^{(\lambda, \mu)} d \Omega_{i} .
$$

Note that these quantities also depend on $T$ through the $T$-dependence of the spectral curve. Another important remark is that such an expression of the Baker-Akhiezer function is available only after selecting a "symplectic homology basis" of the spectral curve, i.e., cycles $A_{I}, B_{I}(I=1, \cdots, g)$ with intersection numbers $A_{I} \cdot A_{J}=B_{I} \cdot B_{J}=0$ and $A_{I} \cdot B_{J}=\delta_{I J}$. (This issue will be discussed in detail in Section 6.)

We now assume that, for a suitable symplectic homology basis, this BakerAkhiezer function coincides with the $\psi$ derived from the leading order equation of multiscale expansion. More precisely, we require their "amplitude part" and "phase part," respectively, to coincide:

$$
\phi^{(0)}=\phi, \quad \frac{\partial S}{\partial T_{i}}=\Omega_{i} .
$$

Actually, the first relation may be relaxed as

$$
\phi^{(0)}=\phi h(T, \lambda)
$$

where $h(T, \lambda)$ is a scalar function independent of $t$ : this takes into account the obvious symmetry

$$
\phi^{(0)} \rightarrow \phi^{(0)} h(T, \lambda)
$$

of the lowest order equation of multiscale expansion. Such a factor $h(T, \lambda)$ might be necessary to proceed to the next order approximation of multiscale analysis. We shall not go into this issue here. 
REMARK. We take this opportunity to correct an error in the previous publication [35]. Substantially the same JWKB ansatz also assumed therein, but with an extra factor of the form $\left(\partial^{2} S / \partial \lambda^{2}\right)^{1 / 2}$ - see Eqs. (13) and (21) therein. This factor itself is nonsense. Fortunately, this does not affect the leading order of multiscale analysis. As mentioned above, however, something like this factor will be necessary in the next order. The arbitrary function $h$ stands for such a compensating factor.

5.3. Modulation equation. Thus, under several assumptions, we have been able to derive a series of relations that link the isomonodromic and isospectral problems. In particular, the following equations are obtained:

$$
\frac{\partial S}{\partial \lambda}=\mu, \quad \frac{\partial S}{\partial T_{i}}=\Omega_{i}
$$

We propose these equations as the modulation equation that governs slow dynamics of the spectral curve

$$
\operatorname{det}\left(M^{(0)}(\lambda)-\mu I\right)=0
$$

Remember that one has to select a symplectic homology basis $A_{I}, B_{I}$ ( $I=$ $1, \cdots, g)$ in order to derive these equations. Actually, selecting a proper homology basis is a non-trivial problem. We discuss this issue in more detail in Section 6, along with a precise characterization of the meromorphic differentials $d \Omega_{i}$.

5.4. Relation to generic Whitham equation. As a piece of evidence that our modulation equation is a reasonable one, we now show that solutions of this equation give a special subfamily of generic Whitham deformations.

The first equation of the modulation equation can be rewritten

$$
d S=\frac{\partial S}{\partial \lambda} d \lambda=\mu d \lambda
$$

In other words, $S$, like $\Omega_{i}$ 's, is the primitive function of a meromorphic differential $d S$ on the spectral curve:

$$
S=\int^{(\lambda, \mu)} \mu d \lambda .
$$

The second part of the modulation equation implies the equations

$$
\frac{\partial}{\partial T_{i}} d S=d \Omega_{i}
$$

Accordingly, the generic Whitham equation

$$
\frac{\partial}{\partial T_{i}} d \Omega_{j}=\frac{\partial}{\partial T_{j}} d \Omega_{j}
$$

follows immediately. (Here, as usual, $\lambda$ is understood to be constant under the Tderivation. Geometrically, this should be treated as a connection. See the paper of Krichever and Phong [59].)

Thus, our modulation equation turns out to yield a special subfamily of generic Whitham deformations. We shall see in Section 8 that an inverse period map yields generals solution of this equation. 
5.5. Possible approach from averaging method. Our heuristic argument has to be justified in a more rigorous form, or at least cross-checked by some other method. A possible check will be to derive the above equations by an averaging argument.

Krichever's averaging method [57, 58] (see also the papers by Fucito et al. [22] Carroll and Chang [60]) seems to be particularly promising. This method employs the so called "dual Baker-Akhiezer function" $\psi^{*}$, i.e., a solution of the dual auxiliary linear problem

$$
\mu \psi^{*}=\psi^{*} M^{(0)}(\lambda), \quad \frac{\partial \psi^{*}}{\partial t_{i}}=\psi^{*} \frac{A_{i}^{(0)}}{\lambda-T_{i}},
$$

along with $\psi$. One can derive, for instance, the following formula evaluating $T$ dependence of the eigenvalue $\mu$ of $M^{(0)}$ :

$$
\frac{\partial \mu}{\partial T_{i}}=\frac{\left\langle\psi^{*} A^{(0)}\left(\lambda-T_{i}\right)^{-2} \psi\right\rangle}{\left\langle\psi^{*} \psi\right\rangle} .
$$

Here $\langle\cdots\rangle$ means the average over the fast variables $t$. This formula can be readily translated to the language of $d S$ (though we have been unable to calculate the average and to identify the resulting equation with the above equation for $d S$ ). Note that the term $A^{(0)}\left(\lambda-T_{i}\right)^{-2}$ in the above formula is the same as the "driving force" term that we encountered in the derivative formula of the characteristic polynomial of $M(\lambda)$.

6. Structure of meromorphic differentials. In this and subsequent sections, we omit the superfix "(0)" and write $A_{i}^{(0)}, M^{(0)}(\lambda)$, etc. as $A_{i}, M(\lambda), \cdots$. This is just for simplifying notations.

6.1. General remarks. The vector-valued Baker-Akhiezer function $\psi$ is single valued on the spectral curve, meromorphic outside the points of $\pi^{-1}\left(\left\{T_{1}, \cdots, T_{N}\right\}\right)$, and has essential singularities of a particular exponential form at these exceptional points. The entries of $\phi$ have a common pole divisor $D$, part of which may overlap with the essential singularities. (In the following, we consider a generic case where this overlapping does not occur.) The essential singularities at points in $\pi^{-1}\left(T_{i}\right)$ are generated by poles of the meromorphic differential $d \Omega_{i}$.

The meromorphic differentials $d \Omega_{i}$ have to be selected for the essential singularities of $\psi$ to match the auxiliary linear problem. This yields conditions on the poles of the meromorphic differentials. Our task in the following is to specify those conditions.

In order to consider this problem, it is convenient to build a matrix solution $\Psi$ of the auxiliary linear problem from the vector-valued Baker-Akhiezer function $\psi$. Let $P_{\alpha}(\lambda)(\alpha=1, \cdots, r)$, denote the points of $\pi^{-1}(\lambda)$. (Of course, also here, such numbering is meaningful only locally.) $\Psi$ is given by

$$
\Psi=\left(\psi\left(P_{1}(\lambda)\right), \cdots, \zeta\left(P_{r}(\lambda)\right)\right)
$$

This matrix has the following factorized form:

$$
\Psi=\Phi \exp \operatorname{diag}\left(\sum t_{i} \Omega_{i}\left(P_{1}(\lambda)\right), \cdots, \sum t_{i} \Omega_{i}\left(P_{r}(\lambda)\right)\right)
$$

$\Phi=\Phi(\lambda)$ is an $r \times r$ matrix originating in the "amplitude part" $\phi$, and invertible at a generic point. 


\subsection{Conditions on meromorphic differentials.}

6.2.1. Poles of $d \Omega_{i}$. All necessary information on poles of $d \Omega_{i}$ can be derived from the following relation, which is obtained by inserting the above expression of $\Psi$ into the auxiliary linear problem:

$$
\frac{\partial \Phi}{\partial t_{i}} \Phi^{-1}+\Phi \operatorname{diag}\left(\Omega_{i}\left(P_{1}(\lambda)\right), \cdots, \Omega_{i}\left(P_{r}(\lambda)\right)\right) \Phi^{-1}=-\frac{A_{i}}{\lambda-T_{i}} .
$$

We now compare the singular part of both hand side at $\lambda=T_{i}$. Since the first term on the left hand side is non-singular, it turns out that $\Omega_{i}$ has a pole of first order at each point of $\pi^{-1}\left(T_{i}\right)$, and the residue is equal to $(-1)$ times an eigenvalues of $A_{i}$. Since the eigenvalues of $A_{i}$ are $\theta_{i \alpha}$, this implies that (after suitably renumbering the eigenvalues) $A_{i}$ can be written

$$
A_{i}=\Phi\left(T_{i}\right) \operatorname{diag}\left(\theta_{i 1}, \cdots, \theta_{i r}\right) \Phi\left(T_{i}\right)^{-1}
$$

and that

$$
\Omega_{i}=-\frac{\theta_{i \alpha}}{\lambda-T_{i}}+\text { non-singular }
$$

in a neighborhood of $P_{\alpha}\left(T_{i}\right)$.

In particular, the meromorphic differential $d \Omega_{i}$ behaves as

$$
d \Omega_{i}=\frac{\theta_{i \alpha}}{\left(\lambda-T_{i}\right)^{2}} d \lambda+\text { non-singular }
$$

in the same neighborhood of $P_{\alpha}\left(T_{i}\right)$. Therefore, if $\theta_{i \alpha} \neq 0, P_{\alpha}\left(T_{i}\right)$ is a pole of $d \Omega_{i}$ of second order. These are all poles that $d \Omega_{i}$ is required to have.

6.2.2. Period integrals of $d \Omega_{i}$. The above conditions on poles determine the meromorphic differential $d \Omega_{i}$ up to a difference of holomorphic differential. We now select a symplectic homology basis $A_{I}, B_{I}(I=1, \ldots, g)$, and put the standard normalization condition

$$
\oint_{A_{I}} d \Omega_{i}=0, \quad I=1, \ldots, g
$$

$d \Omega_{i}$ is thus uniquely determined.

6.3. Back to modulation equation. This is the end of the precise description of our modulation equation. Let us finally reconfirm the roles of the three sets of parameters $\theta_{i \alpha}, T_{i}$ and $h_{m \ell}$ in the defining equation of the spectral curve:

- The coadjoint orbit invariants $\theta_{i \alpha}$ are constant parameters.

- The positions of poles $T_{i}$ are "time variables."

- The isospectral invariants $h_{m \ell}$ are "dynamical variables."

6.4. Remarks on symplectic homology basis. The choice of symplectic homology basis is a non-trivial problem. The work of Flaschka, Forest and McLaughlin [30] provides a typical example for considering this problem. In their work, the meromorphic differentials $d \Omega_{i}$ are normalized along the so called " $\mu$-cycles." These cycles are homologous to trajectories of the "auxiliary spectrum," which are nothing but 
the degree $g$ divisor $\sum_{i=1}^{g}\left(\lambda_{i}, \zeta_{i}\right)$ of zeros of a Baker-Akhiezer function on the $\mathrm{KdV}$ hyperelliptic spectral curve

$$
\zeta^{2}=\prod_{i=1}^{2 g+1}\left(\lambda-e_{i}\right)
$$

The Whitham averaging over the quasi-periodic motion on the Jacobian variety eventually boils down to period integrals along these trajectories. As this typical example shows clearly, the normalization condition of $d \Omega_{i}$ is by no means arbitrary, but determined by the geometric structure of motion of a divisor on the spectral curve. (In fact, as Ercolani, Forest and McLaughlin noted [61, 62], this issue is already considerably delicate in the case of the sine-Gordon equation.)

Meanwhile, our formulation of the modulation equation itself works for any choice of the symplectic homology basis. Furthermore, the results of the subsequent sections also hold irrespective of the choice of the symplectic homology basis. Presumably, it is only a subset of solutions of our modulation equation that actually correspond to the true modulational description of isomonodromic problems. This issue, too, forms part of the hard analytical problems that we do not pursue in this paper.

\section{Solutions of modulation equation.}

7.1. Period integrals à la Seiberg-Witten. In the following, let $h=\left\{h_{I} \mid\right.$ $I=1, \cdots, g\}$ denote the $g$-tuple of isospectral invariants $h_{m \ell}(\ell=2, \cdots, r, m=$ $0, \cdots, \delta_{\ell}-1$ ) (see Section 3). With the other parameters $\theta_{i \alpha}$ and $T_{i}$ being fixed, the spectral curve forms a $g$-dimensional deformation family parametrized by these isospectral invariants. This is the same situation as the Seiberg-Witten solution and its various generalizations [36]-[47]. One can indeed derive the following analogous results.

- A set of (local) coordinates on the $g$-dimensional moduli space of spectral curves are given by the period integrals

$$
a_{I}=\oint_{A_{I}} d S, \quad I=1, \cdots, g
$$

Here (and in the following), $A_{I}, B_{I}(I=1, \cdots, g)$ are the same symplectic homology basis as used in the definition of the modulation equation.

- Another set of (local) coordinates are given by the dual period integrals

$$
b_{I}=\oint_{B_{I}} d S, \quad I=1, \cdots, g .
$$

In the Seiberg-Witten theory, they are denoted by $a_{I}^{D}$.

- There is a (locally defined) function $\mathcal{F}=\mathcal{F}(a)$ ("prepotential") of $a=\left\{a_{I}\right\}$ such that

$$
\frac{\partial \mathcal{F}}{\partial a_{I}}=b_{I}
$$

- The second derivatives of $\mathcal{F}$ coincide with matrix elements of the period matrix,

$$
\frac{\partial^{2} \mathcal{F}}{\partial a_{I} \partial a_{J}}=\mathcal{T}_{I J}=\oint_{B_{I}} d \omega_{J}
$$


where $d \omega_{I}(I=1, \cdots, g)$ are a basis of holomorphic differentials uniquely determined by the normalization condition

$$
\oint_{A_{I}} d \omega_{J}=\delta_{I J}
$$

In a sense, the rest of this paper is devoted to verifying these results in an $T$-dependent form.

The goal of this section is to show that the inverse period map $a \mapsto h$ solves our deformation equation. We first establish the invertibility of the period map $h \mapsto a$. The inverse map $a \mapsto h$ then turns out to satisfy a deformation equation of SeibergWitten type (with respect to $a_{i}$ 's), as well as our modulation equation (with respect to $T_{i}$ 's). Most results and proofs are a rather straightforward generalization of those of the Seiberg-Witten solution [36]-[47].

It should be added that Krichever and Phong [59] gave a similar period map construction of Whitham deformations in a far more general form. Our case can also be treated as a special case of their results. Nevertheless we dare to show the following construction, because this will be a simpler and more explicit proof in the present special setup.

The notion of prepotential will be discussed in detail in Section 8.

7.2. Invertibility of period map. This subsection is organized as follows. Firstly, we construct a basis $d \tilde{\omega}_{I}(I=1, \cdots, g)$ of holomorphic differentials as derivatives of $d S$ with respect to $h_{I}$ 's. Secondly, we examine the linear relations between this basis and the normalized basis $d \omega_{I}(I=1, \cdots, g)$. As a corollary, (local) invertibility of the period map $h \rightarrow a$ follows.

7.2.1. Basis of holomorphic differentials. We here consider $\mu$ to be a (multivalued) function $\mu=\mu(T, h, \lambda)$ of $T, h$ and $\lambda$, and differentiate $d S(T, h, \lambda)=$ $\mu(T, h, \lambda) d \lambda$ by $h_{I}$. This gives the differential

$$
d \tilde{\omega}_{I}=\frac{\partial}{\partial h_{I}} d S=\frac{\partial \mu(T, h, \lambda)}{\partial h_{I}} d \lambda .
$$

We now show that this gives a basis of holomorphic differentials on the spectral curve. The following reasoning is borrowed from the work of Adams, Harnad and Hurtubise [10].

This definition of $d \tilde{\omega}_{I}$ can be cast into a more tractable form as follows. First rewrite it in terms of $\tilde{\mu}=f(\lambda) \mu$ (see Section 3):

$$
d \tilde{\omega}_{I}=\frac{1}{f(\lambda)} \frac{\partial \tilde{\mu}(T, h, \lambda)}{\partial h_{I}} d \lambda .
$$

Differentiating the equation

$$
\tilde{F}(\lambda, \tilde{\mu})=\operatorname{det}(f(\lambda) M(\lambda)-\mu I)=0
$$

of the spectral curve in the $(\lambda, \tilde{\mu})$ coordinates gives

$$
\frac{\partial \tilde{\mu}}{\partial h_{I}}=-\frac{\partial \tilde{F}(\lambda, \tilde{\mu}) / \partial h_{I}}{\partial \tilde{F}(\lambda, \tilde{\mu}) / \partial \tilde{\mu}} .
$$


Now recall that $h_{I}$ is just an abbreviation of one of $h_{m \ell}$ 's in the following formula of Section 3:

$$
\tilde{F}(\lambda, \tilde{\mu})=\tilde{F}^{0}(\lambda, \tilde{\mu})+f(\lambda) \sum_{\ell=2}^{r} \sum_{m=0}^{\delta_{\ell}} h_{m \ell} \lambda^{m} \tilde{\mu}^{r-\ell} .
$$

Therefore, for $h_{I}=h_{m \ell}$,

$$
\frac{\partial \tilde{F}(\lambda, \tilde{\mu})}{\partial h_{m \ell}}=f(\lambda) \lambda^{\ell} \tilde{\mu}^{r-m} .
$$

Thus, eventually, we obtain the following expression of $d \tilde{\omega}_{I}=d \tilde{\omega}_{m \ell}$ :

$$
d \tilde{\omega}_{m \ell}=-\frac{\lambda^{\ell} \tilde{\mu}^{r-m}}{\partial \tilde{F}(\lambda, \tilde{\mu}) / \partial \mu} d \lambda
$$

The last expression may be viewed as the Poincare residue of a meromorphic 2 form on the $(\lambda, \tilde{\mu})$ plane with pole divisor along the spectral curve. Since $2 \leq \ell \leq r$ and $0 \leq m \leq \delta_{\ell}-1$, a standard argument leads to the following result:

Proposition 7.1. The differentials d $\tilde{\omega}_{I}(I=1, \cdots, g)$, form a basis of holomorphic differentials on the spectral curve.

7.2.2. Linear relations between two bases. Since $d \tilde{\omega}_{I}$ and $d \omega_{I}$ both give a basis of holomorphic differentials, they should be linked by an invertible linear transformation:

$$
d \tilde{\omega}_{I}=\sum_{J=1}^{g} \mathcal{A}_{I J} d \omega_{J}
$$

The matrix $\mathcal{A}=\left(\mathcal{A}_{I J}\right)$ of the coefficients is invertible.

In fact, the matrix $\mathcal{A}$ is given by the period integrals

$$
\mathcal{A}_{I J}=\oint_{A_{J}} d \tilde{\omega}_{I}
$$

as one can readily see by integrating the above linear relation of $d \tilde{\omega}_{I}$ and $d \omega_{J}$ along $A_{J}$. Similarly, integrating along $B_{J}$ yields the matrix relation

$$
\mathcal{B}=\mathcal{A T}
$$

where the matrix elements of $\mathcal{B}=\left(\mathcal{B}_{I J}\right)$ are given by

$$
\mathcal{B}_{I J}=\oint_{B_{J}} d \tilde{\omega}_{I}
$$

In particular, the period matrix $\mathcal{T}$ can be written

$$
\mathcal{T}=\mathcal{A}^{-1} \mathcal{B}
$$


7.2.3. Invertibility of period map. We now show the following result, which shows that the matrix is in fact the Jacobian matrix of the period map $h \mapsto a$.

PROPOSITION 7.2.

$$
\mathcal{A}_{I J}=\frac{\partial a_{J}}{\partial h_{I}}
$$

Proof. $a_{J}$ is defined by the period integral

$$
a_{J}=\oint_{A_{J}} d S
$$

Its $h_{I}$-derivative can be calculates as follows:

$$
\frac{\partial a_{J}}{\partial h_{I}}=\oint_{A_{J}} \frac{\partial}{\partial h_{I}} d S=\oint_{A_{J}} d \tilde{\omega}_{I}=\mathcal{A}_{I J}
$$

Since $\mathcal{A}$ is an invertible matrix, we have:

COROLlaRY 7.3. The period map $h \mapsto a$ is (locally) invertible.

7.3. Solving deformation equations by inverse period map. We now take into account the variables $T=\left\{T_{i}\right\}$, and consider the $I$-th coordinate of the inverse map $a \mapsto h$ as a function of $T$ and $a$ :

$$
h_{I}=h_{I}(T, a) .
$$

(In order to obtain these functions, one has to solve the defining equation of the $a_{I}$ 's for the parameters $h_{I}$. This is by no means an easy task.) Accordingly, $d S=\mu d \lambda$ becomes a meromorphic differential depending on the parameters $(T, a)$ :

$$
d S=d S(T, a)
$$

We can now prove that $d S$ satisfies the modulation equation with respect to $T$ :

Proposition 7.4. dS satisfies the modulation equation

$$
\frac{\partial}{\partial T_{i}} d S=d \Omega_{i}
$$

Proof. Let us tentatively define

$$
d \tilde{\Omega}_{i}=\frac{\partial}{\partial T_{i}} d S=\frac{\partial \mu}{\partial T_{i}} d \lambda
$$

We show that this differential satisfies all conditions that characterize $d \Omega_{i}$. By the uniqueness, then, $d \tilde{\Omega}_{i}$ coincides with $d \Omega_{i}$, and the proof is completed.

(i) Locations of poles of $d \tilde{\Omega}_{i}$. Differentiating the equation of the spectral curve

$$
F(\lambda, \mu)=\operatorname{det}(M(\lambda)-\mu I)=0
$$

gives the relation

$$
\frac{\partial \mu}{\partial T_{i}}=-\frac{\partial F(\lambda, \mu) / \partial T_{i}}{\partial F(\lambda, \mu) / \partial \mu}
$$


Therefore, apart from the poles of $\mu$, the derivative $\partial \mu / \partial T_{i}$ can have poles at the zeros of $\partial F / \partial \mu$ (i.e., at ramified points of $\pi$ ). Let $e$ be the ramification index at a ramified point. $\partial \mu / \partial T_{i}$ has a pole of order at most $e-1$ there. This pole, however, is canceled by zeros of $d \lambda$ because $d \lambda$ has a zero of order $e-1$ at the same point. Meanwhile, in a neighborhood of each point of $\pi^{-1}(\infty)$,

$$
\frac{\partial \mu}{\partial T_{i}}=\left(\theta_{\infty \alpha} \lambda^{-2}+O\left(\lambda^{-2}\right)\right) d \lambda
$$

so that the derivative $\partial \mu / \partial T_{i}$ cancels the second order poles of $d \lambda$ at these points. Thus, poles of $d \tilde{\Omega}_{i}$ are limited to points in $\pi^{-1}\left(\left\{T_{1}, \cdots, T_{N}\right\}\right)$.

(ii) Singular behavior of $d \tilde{\Omega}_{i}$ at poles. We examine the singular behavior of $d \tilde{\Omega}_{i}$ at each point of $\pi^{-1}\left(T_{j}\right)=\left\{P_{j 1}, \cdots, P_{j r}\right\}$. Recall that, in a neighborhood of $P_{i \alpha}, \mu$ behaves as

$$
\mu=-\frac{\theta_{i \alpha}}{\lambda-T_{j}}+\text { non-singular. }
$$

Therefore

$$
\frac{\partial \mu}{\partial T_{i}}= \begin{cases}\text { non-singular } & (j \neq i) \\ -\frac{\theta_{i \alpha}}{\left(\lambda-T_{i}\right)^{2}}+\text { non-singular } & (j=i)\end{cases}
$$

Consequently, $d \tilde{\Omega}_{i}$ has poles only at the points over $\lambda=T_{i}$, and exhibits there the same singular behavior as $d \Omega_{i}$ does.

(iii) Period Integrals of $d \tilde{\Omega}_{i}$. Since the deformations leave $a_{I}$ 's invariant, we have

$$
\oint_{A_{I}} \frac{\partial}{\partial T_{i}} d S=\frac{\partial}{\partial T_{i}} \oint_{A_{I}} d S=\frac{\partial a_{I}}{\partial T_{i}}=0 .
$$

Thus $d \tilde{\Omega}_{i}$ satisfies the normalization condition of periods, too.

Similarly, $d S$ turns out to satisfy a deformation equation of Seiberg-Witten type with respect to $a_{I}$ 's:

Proposition 7.5. dS satisfies the deformation equation

$$
\frac{\partial}{\partial a_{I}} d S=d \omega_{I}, \quad I=1, \cdots, g
$$

of Seiberg-Witten type.

Proof. This is just a consequence of the chain rule of differentiation:

$$
\frac{\partial}{\partial a_{I}} d S=\sum_{J} \frac{\partial h_{J}}{\partial a_{I}} \frac{\partial}{\partial h_{J}} d S=\sum_{J}\left(\mathcal{A}^{-1}\right)_{I J} d \tilde{\omega}_{J}=d \omega_{I}
$$

Thus, we have been able to show that the inverse period map $a \mapsto h$ solves the coupled deformation equations

$$
\frac{\partial}{\partial T_{i}} d S=d \Omega_{i}, \quad \frac{\partial}{\partial a_{I}} d S=d \omega_{I} .
$$

(Reconfirm, once again, that the differentiation is understood to leave $\lambda$ constant, i.e., $\partial \lambda / \partial T_{i}=\partial \lambda / \partial a_{I}=0$.) 
7.4. Remarks on structure of $d S$. It seems remarkable that although our meromorphic differential $d S$ has simple poles with non-zero residues, their derivatives in deformation variables are holomorphic differentials or meromorphic differentials of second kind (i.e., meromorphic differentials with higher order poles and no residue). This is reminiscent of the structure of the Seiberg-Witten meromorphic differential in supersymmetric gauge theories coupled with matter fields [36]-[47].

Because of this fact, $d S$ cannot be written as a linear combination of $d \omega_{I}$ and $d \Omega_{i}$; we need extra meromorphic differentials of the third kind, say $d \Pi$, with the same singularity structure as $d S$ but with vanishing $A_{I}$-cycles. $d S$ can be written

$$
d S=\sum_{I=1}^{g} a_{I} d \omega_{I}+d \Pi
$$

Of course $d \Pi$ can be further decomposed into a sum of more elementary meromorphic differentials, but can never be a linear combination of $d \Omega_{i}$ 's.

\section{Prepotential.}

8.1. Definition of prepotential. The notion of prepotential has been formulated in a quite general framework by Krichever [31, 32] and Dubrovin [33, 33]. Following their formalism we can now define a prepotential $\mathcal{F}=\mathcal{F}(T, a)$ by the following differential equation.

Proposition 8.1. The following differential equations is integrable in the sense of Frobenius:

$$
\frac{\partial \mathcal{F}}{\partial a_{I}}=\oint_{B_{I}} d S, \quad \frac{\partial \mathcal{F}}{\partial T_{i}}=\sum_{\alpha=1}^{r} \operatorname{Res}_{P_{i \alpha}} \frac{\theta_{i \alpha}}{\lambda-T_{i}} d S
$$

Here $P_{i \alpha}, \alpha=1, \cdots, r$, denote the points of $\pi^{-1}\left(T_{i}\right)$ such that

$$
\mu=\frac{\theta_{i \alpha}}{\lambda-T_{i}}+\text { non-singular }
$$

in a neighborhood of $P_{i \alpha}$.

8.2. Equivalent definition. Before proving the above result, we note here that the defining equations of $\mathcal{F}$ can be rewritten in the following more compact form:

$$
\frac{\partial \mathcal{F}}{\partial a_{I}}=b_{I}, \quad \frac{\partial \mathcal{F}}{\partial T_{i}}=H_{i}
$$

The equivalence of the first equation is obvious from the definition of $b_{I}$. Let us verify the equivalence of the second equation in some detail. We first rewrite the definition of $H_{i}$ into a contour integral

$$
H_{i}=\frac{1}{2 \pi \sqrt{-1}} \oint_{\left|\lambda-T_{i}\right|=\delta} \frac{1}{2} \operatorname{Tr} M(\lambda)^{2} d \lambda
$$

along a sufficiently small circle (of radius $\delta$ ) around $\lambda=T_{i}$. Let $\mu_{\alpha}, \alpha=1, \cdots, r$, denote the eigenvalues of $M(\lambda)$; they correspond to the $r$ sheets of the spectral curve in a neighborhood of $\pi^{-1}\left(T_{i}\right)$. Expressing the trace of $M(\lambda)^{2}$ in terms of these eigenvalues, 
we can rewrite the above integral formula as:

$$
\begin{aligned}
H_{i} & =\frac{1}{2 \pi \sqrt{-1}} \oint_{\left|\lambda-T_{i}\right|=\delta} \sum_{\alpha=1}^{r} \frac{\mu_{\alpha}^{2}}{2} d \lambda \\
& =\sum_{\alpha=1}^{r} \frac{1}{2 \pi \sqrt{-1}} \oint_{C_{i_{\alpha}}} \frac{\mu^{2}}{2} d \lambda .
\end{aligned}
$$

Now recall that $\mu$ has a Laurent expansion of the form

$$
\mu=\frac{\theta_{i \alpha}}{\lambda-T_{i}}+c_{i \alpha, 0}+c_{i \alpha, 1}\left(\lambda-T_{i}\right)+\cdots
$$

in a neighborhood of $P_{i \alpha}$. From this fact, we can easily derive the relation

$$
\oint_{C_{i \alpha}} \frac{\mu^{2}}{2} d \lambda=\oint_{C_{i \alpha}} \frac{\theta_{i \alpha}}{\lambda-T_{i}} d S
$$

This relation and the above expression of $H_{i}$ give

$$
H_{i}=\sum_{\alpha=1}^{r} \operatorname{Res}_{P_{i \alpha}} \frac{\theta_{i \alpha}}{\lambda-T_{i}} d S .
$$

This leads to the second expression of the defining equation of the prepotential.

8.3. Proof of proposition. The idea of proof is the same as the standard one based on Riemann's bilinear relation. Integrability conditions to be checked are the following:

$$
\frac{\partial H_{j}}{\partial T_{i}}=\frac{\partial H_{i}}{\partial T_{j}}, \quad \frac{\partial H_{j}}{\partial a_{I}}=\frac{\partial b_{I}}{\partial T_{j}}, \quad \frac{\partial b_{J}}{\partial a_{I}}=\frac{\partial b_{I}}{\partial a_{J}} .
$$

Here $b_{I}, b_{J}, H_{i}$ and $H_{j}$ are used for simplifying notations; in the verification below, we have to return to the integral expressions of these quantities.

These three relations can be verified in much the same way. We show the derivation of the second relation in detail. First, differentiating the integral formula

$$
H_{j}=\sum_{\alpha} \frac{1}{2 \pi \sqrt{-1}} \oint_{C_{j \alpha}} \frac{\theta_{j \alpha}}{\lambda-T_{j}} d S
$$

by $a_{I}$ gives

$$
\frac{\partial H_{j}}{\partial a_{I}}=\sum_{\alpha} \frac{1}{2 \pi \sqrt{-1}} \oint_{C_{j \alpha}} \frac{\theta_{j \alpha}}{\lambda-T_{j}} d \omega_{I} .
$$

Similarly, from the integral formula of $b_{I}$,

$$
\frac{\partial b_{I}}{\partial T_{j}}=\oint_{B_{I}} d \Omega_{j} .
$$

By Riemann's bilinear relation, $d \Omega_{j}$ and $d \omega_{I}$ satisfies the relation

$$
\text { (*) } \frac{1}{2 \pi \sqrt{-1}} \oint_{\partial \Delta} \Omega_{j} d \omega_{I}=\sum_{J}\left(\oint_{A_{K}} d \Omega_{j} \oint_{B_{K}} d \omega_{I}-\oint_{B_{K}} d \Omega_{j} \oint_{A_{K}} d \omega_{I}\right) \text {. }
$$


Here $\Delta$ is a simply connected surface with boundary ( $4 g$-gon) obtained in a standard way by cutting the Riemann surface of the spectral curve along $2 g$ paths. The boundary $\partial \Delta$ is oriented in the direction encircling interior points anti-clockwise. We now evaluate both hand sides. As for the left hand side of $(*), \Omega_{j}$ has poles at the points $P_{j 1}, \cdots, P_{j r}$ in $\Delta$ whereas $d \omega_{I}$ has of course no pole. Therefore the contour integral along $\partial \Delta$ splits into a sum of contour integrals along $C_{j 1}, \cdots, C_{j r}$. Furthermore, the singular behavior of $\Omega_{j}$ in a neighborhood of $P_{j \alpha}$ is such that

$$
\Omega_{j}=-\theta_{j \alpha} /\left(\lambda-T_{j}\right)+\text { non-singular. }
$$

Therefore

$$
\text { LHS of }(*)=-\sum_{\alpha} \frac{1}{2 \pi \sqrt{-1}} \oint \frac{\theta_{j \alpha}}{\lambda-T_{j}} d \omega_{I} \text {. }
$$

Now consider the the right hand side of $(*)$. The integrals of $d \Omega_{j}$ along $A_{K}$ 's all vanish by the normalization conditions of $d \Omega_{j}$. Its integrals along $B_{K}$ 's do not vanish in general, but now the integral of $d \omega_{I}$ along $A_{K}$ 's vanish except for $K=I$; in the case of $K=I$, the latter integral is equal to 1 by the normalization conditions of $d \omega_{I}$. Thus

$$
\text { RHS of }(*)=-\oint_{B_{I}} d \Omega_{j}
$$

These relations imply the second integrability condition.

In the verification of the first integrability condition, it is convenient to express $d \Omega_{i}$ as a sum of meromorphic differentials $d \Omega_{i \alpha}$ with just one pole at $P_{i \alpha}$ and normalized by the same condition of vanishing $A$-periods. The integrability condition can then be reduced to Riemann's bilinear relation for $d \Omega_{i \alpha}$ and $d \Omega_{j \beta}$.

The third integrability condition is the easiest to verify. This case, however, is the most interesting in the context of the Seiberg-Witten theory. Differentiating $b_{J}$ now by $a_{I}$ gives

$$
\frac{\partial b_{J}}{\partial a_{I}}=\oint_{B_{J}} d \omega_{I}=\mathcal{T}_{I J}
$$

This is equal to $\partial b_{I} / \partial a_{J}$, because the period matrix $\mathcal{T}$ is symmetric. (One can also directly deduce this conclusion by applying Riemann's bilinear relation to $d \omega_{I}$ and $d \omega_{J}$. This is indeed a usual proof of the relation $\mathcal{T}_{I J}=\mathcal{T}_{J I}$ !)

This completes the proof of the proposition.

As a corollary of the proposition and the final part of the proof, we obtain the following result:

COROLlaRY 8.2. The second a-derivatives of $\mathcal{F}=\mathcal{F}(T, a)$ coincide with the matrix elements of the period matrix:

$$
\frac{\partial^{2} \mathcal{F}}{\partial a_{I} \partial a_{J}}=\mathcal{T}_{I J}
$$

Thus our treatment of the prepotential incorporates all essential aspects of the prepotential of Seiberg-Witten type. 
8.4. Final remarks. (i) The definition of prepotential shows a link with the notion of $\tau$ function. Recall that $H_{i}$ is equal to a logarithmic derivative of the $\tau$ function of the Schlesinger equation. In the $\epsilon$-dependent formulation,

$$
H_{i}=\epsilon \frac{\partial \log \tau}{\partial T_{i}} .
$$

Note, however, that $\log \tau$ and $\mathcal{F}$ are by no means in a simple proportional relation, because some averaging operation intervenes.

(ii) Unlike the prepotentials in topological conformal field theories and the SeibergWitten theory, our prepotential $\mathcal{F}$ seems to possess no manifest homogeneity of degree two. This issue seems to be linked with the unusual structure of $d S$ that we pointed out in the end of the last section. Presumably our prepotential (and the modulation equation) will have hidden homogeneity, which emerges after introducing more deformation variables.

9. Conclusion. The body of this paper consists of two part. The first part is concerned with the derivation of the modulation equation. This equation is expected to describe slow dynamics of the spectral curve in isospectral approximation, in the sense of Garnier, of the ( $\epsilon$-dependent) Schlesinger equation. Although our method for deriving this modulation equation is heuristic, we believe that this gives a correct answer (under a suitable choice of the symplectic homology basis). The second part of this paper is devoted to a complete description of solutions of the modulation equation, as well as the notion of prepotential. We have been able to obtain the following fundamental results:

- General solutions are obtained by an inverse period map. The period map is given by period integrals of Seiberg-Witten type.

- The modulation equation can coexist with a deformation equation of SeibergWitten type. They altogether form a commuting set of flows on the $g$ dimensional moduli space of spectral curves.

- A prepotential can be defined on this extended commuting flows. This construction is parallel to already known examples of prepotentials.

These results, too, strongly support the validity of the modulation equation.

A number of problems are left unanswered. The most crucial is of course the issue of validity of our derivation of modulation equation. We have presented a few fragmental ideas in this direction. Krichever's averaging method seems to be the most powerful and universal approach to this problem; we, however, have no idea how to calculate the averaged quantities to obtain period integrals. Presumably, Vereschagin's method will be a hint to this issue. Also, we would like to stress that generalizing the averaging calculation of Flaschka, Forest and McLaughlin to nonhyperelliptic spectral curves is still a very important problem; the spectral Darboux coordinates of the Montreal group should be used in place of the classical auxiliary spectrum.

The methods presented in this paper will be generalized to other types of isomonodromic problems. An immediate, but also interesting generalization is the case with irregular singular points. For instance, if an irregular singular point of Poincaré rank one is added to $\lambda=\infty$, the resulting isomonodromic problem becomes the so called JMMS equation [48]. This equations exhibits a remarkable "duality" [12], which will be inherited to the modulation equation. This issue is discussed in a separate paper [63]. An even more interesting direction is a generalization to isomonodromic problems on an elliptic (and higher genus) Riemann surface. Examples of such isomonodromic 
problems have been constructed by Okamoto's group in a geometric framework [64][69]. Recently, Levin and Olshanetsky presented another framework based on the method of Hamiltonian reduction, and pointed out a link with the Whitham equation $[70,71]$.

\section{REFERENCES}

[1] R. FuCHS, Über lineare homogene Differentialgleichungen zweiter ordnung mit drei in Endlich gelegene wesentlich singulären Stellen, Math. Ann., 63 (1907), pp. 301-321.

[2] R. GARNiER, Sur des équations différentielles du troisième ordre dont l'intégral générale et uniforme et sur une class d'équations nouvelles dódre supérieur dont l'intégrale générale a ses points critiques fixes, Ann. Sci. École Norm. Sup., 29:3 (1912), pp. 1-126.

[3] L. Schlesinger, Über eine Klasse von Differentialsystemen beliebiger Ordnung mit festen kritischen Punkten, J. für Math., 141 (1912), pp. 96-145.

[4] R. GARNIER, Solution du problème de Riemann pour les systèmes différentiels liéaires du second ordre, Ann. Sci. École Norm. Sup., 43:3 (1926), pp. 177-307.

[5] - Etude de l'intégrale générale de l'équation VI de M. Painlevé dans le voisinage de ses singularité transcendantes, Ann. Sci. École Norm. Sup., 34:3 (1917), pp. 239-353.

[6] J. Moser, Geometry of quadrics and spectral theory, in Chern Symposium, Berkeley, 1979, Springer-Verlag, Berlin, Heidelbeg, New York, 1980, pp. 147-188.

[7] D. Mumford, Tata lectures on theta, vol. II, Birkhäuser, Boston, 1983.

[8] M.R. Adams, J. HaRnad and E. Previato, Isospectral Hamiltonian flows in finite and infinite dimensions, I, Commun. Math. Phys., 117 (1988), pp. 451-500.

[9] M.R. Adams, J. HaRnad And J. HuRtubise, Isospectral Hamiltonian flows in finite and infinite dimensions, II, Commun. Math. Phys., 134 (1990), pp. 555-585.

[10] - Darboux coordinates and Liouville-Arnold integration in loop algebras, Commun. Math. Phys., 155 (1993), pp. 385-413.

[11] A. BeAuville, Jacobiennes des courbes spectrales et systèmes hamiltoniens complètement intégrables, Acta Math., 164 (1990), pp. 211-235.

[12] J. HARnAD, Dual isomonodromic deformations and moment maps to loop algebras, Commun. Math. Phys., 166 (1994), pp. 337-365.

[13] J. Harnad, C.A. Tracy AND H. Widom, Hamiltonian structure of equations appearing in Random matrices, in: Low Dimensional Topology and Quantum Field Theory (ed. H. Osborn), pp. 231-245 (Plenum, New York, 1993).

[14] J. HARNAD AND M. ROUThIER, R-matrix construction of electromagnetic models for Painlevé transcendents, J. Math. Phys., 36 (1995), pp. 4863-4881.

[15] J. HARNAD AND M.-A. Wisse, Loop algebra moment maps and Hamiltonian models for the Painlevé transcendents, in Mechanics Day workshop Proceedings (eds. P.S. Krishnaprasad et al.), AMS-Fields Inst. Commun., 7 (1996), pp. 155-169.

[16] P. Boutroux, Recherches sur les transcendantes des M. Painlevé et l'étude asymptotique des équations différentielles du second ordre, Ann. Sci. Ecole Norm. Sup., 30:3 (1913), pp. $255-376$.

[17] H. FlaschKa and A. Newell, Monodromy- and spectrum-preserving deformations, I, Commun. Math. Phys., 76 (1980), pp. 65-116.

[18] - Multiphase similarity solutions of integrable evolution equations, Physica D, 3 (1981), pp. 203-221.

[19] G. Moore, Matrix models of $2 D$ gravity and isomonodromic deformations, Prog. Theor. Phys. Suppl., 102 (1990), pp. 255-285.

[20] S.P. NoviKov, Quantization of finite-gap potentials potentials and nonlinear quasiclassical approximation in nonperturbative string theory, Funct. Anal. Appl., 24 (1990), pp. 296306.

[21] I.M. Krichever, On Heisenberg relations for the ordinary linear differential operators, ETH Zürich preprint, June 1990.

[22] F. Fucito, A. Gamba, M. Martellini and O. Ragnisco, Nonlinear WKB analysis of the string equation, Int. J. Mod. Phys. B, 6 (1992), pp. 2123-2148.

[23] V.L. Vereschagin, Asymptotics of solutions of the discrete string equation, Physica D, 95 (1996), pp. 268-282.

[24] G.B. Whitham, Linear and Nonlinear Waves, Wiley-Interscience, New York, 1974.

[25] S.Yu. DobroкhотоV AND V.P. MASLOV, Finite-zone, almost-periodic solutions in WKB approximations, J. Soviet Math., 16:6 (1981), pp. 1433-1487. 
[26] B.A. Dubrovin, V.B. Matveev And S.P. Novikov, Nonlinear equations of Korteweg-de Vries type, finite band operators and Abelian varieties, Russian Math. Surveys, 31:1 (1976), pp. 59-146.

[27] I.M. KRICHEVER, Methods of algebraic geometry in the theory of nonlinear equations, Russian Math. Surveys, 32:6 (1977), pp. 185-214.

[28] - Integration of nonlinear equations by the method of algebraic geometry, Funct. Anal. Appl., 11 (1977), pp. 12-26.

[29] B.A. Dubrovin AND S.P. Novikov, Hydrodynamics of weakly deformed soliton lattices: Differential geometry and Hamiltonian theory, Russian Math. Surveys, 44:6 (1989), pp. 35-124.

[30] H. FlaschKa, M.G. Forest AND D.W. MCLAUGhlin, Multiphase averaging and the inverse spectral solution of the Korteweg-de Vries equation, Comm. Pure Appl. Math., 33 (1980) pp. 739-784.

[31] I.M. Krichever, The dispersionless Lax equations and topological minimal models, Commun. Math. Phys., 143 (1992), pp. 415-429.

[32] - The $\tau$-function of the universal Whitham hierarchy, matrix models and topological field theories, Comm. Pure. Appl. Math., 47 (1994), pp. 437-475.

[33] B.A. Dubrovin, Hamiltonian formalism of Whitham hierarchies and topological LandauGinzburg models, Commun. Math. Phys., 145 (1992), pp. 195-207;

[34] — Geometry of 2D topological field theories, in Lecture Notes in Math. 1620, SpringerVerlag, Berlin, Heidelbeg, New York, 1996, pp. 120-348.

[35] K. TAKASAKI AND T. NAKATSU, Isomonodromic deformations and supersymmetric Yang-Mills theories, Int. J. Mod. Phys. A, 11:31 (1996), pp. 5505-5518.

[36] A. Gorsky, I. Krichever, A. Marshakov, A. Mironov and A. Morozov, Integrability and exact Seiberg-Witten solution, Phys. Lett. B, 335 (1995), pp. 466-474.

[37] E. MARTINEC AND N. WARnER, Integrable systems and supersymmetric gauge theories, Nucl. Phys. B, 459 (1996), pp. 97-112.

[38] T. NAKATSU AND K. TAKASAKI, Whitham-Toda hierarchy and $N=2$ supersymmetric YangMills theory, Mod. Phys. Lett. A, 11 (1996), pp. 157-168.

[39] R. Donagi and E. Witten, Supersymmetric Yang-Mills theory and integrable systems, Nucl. Phys. B, 460 (1996), pp. 299-334.

[40] T. Eguchi And S.-K. YAng, Prepotentials of $N=2$ supersymmetric gauge theories and soliton equations, Mod. Phys. Lett. A, 11 (1996), pp. 131-138.

[41] E. MARTINEC, Integrable structures in supersymmetric gauge and string theory, Phys. Lett. B, 367 (1996), pp. 91-96.

[42] A. GORSKY AND A. MARShaKov, Towards effective topological theories on spectral curves, Phys. Lett. B, 375 (1996), pp. 127-134.

[43] H. Itroyama AND A. Morozov, Integrability and Seiberg-Witten theory. Curves and periods, Nucl. Phys. B, 477 (1996), pp. 855-877.

[44] H. Itoyama And A. Morozov, Prepotential and the Seiberg-Witten theory, Nucl. Phys. B, 491 (1997), pp. 529-573.

[45] A. Marshakov, Exact solutions to quantum field theories and integrable equation, Mod. Phys. Lett. A, 11 (1996), pp. 1169-1184.

[46] C. AHN AND S. NAM, Integrable structure in supersymmetric gauge theories with massive hypermultiplets, Phys. Lett. B, 387 (1996), pp. 304-309.

[47] A. Marshakov, On integrable systems and supersymmetric gauge theories, Theor. Math. Phys., 112 (1997), pp. 791-826.

[48] M. Jimbo, T. Miwa, Y. Môri And M. Sato, Density matrix of an impenetrable Bose gas and the fifth Painlevé transcendents, Physica D, 1 (1980), pp. 80-158.

[49] M. Jimbo, T. Miwa AND K. UEno, Monodromy preserving deformation of linear ordinary differential equations with rational coefficients, I, Physica D, 2 (1981), pp. 306-352.

[50] M. Jimbo AND T. MiWA, Monodromy preserving deformation of linear ordinary differential equations with rational coefficients, II, Physica D, 2 (1981), pp. 407-448.

[51] — Monodromy preserving deformation of linear ordinary differential equations with rational coefficients, III, Physica D, 4 (1981), pp. 26-46.

[52] V.L. Vereschagin, Nonlinear quasiclassics and Painlevé equations, hep-th/9605092.

[53] К. Окамото, Isomonodromic deformations and Painlevé equations, and the Garnier system, J. Fac. Sci. Univ. Tokyo, Sect. IA, Math., 33 (1986), pp. 575-612.

[54] A. Newell And J. Whitehead, Finite bandwidth, finite amplitude convection, J. Fluid Mech., 38 (1969), pp. 279-303.

[55] J.D. Gibbon AND M.J. MCGuiness, Amplitude equations at the critical points of unstable dispersive physical systems, Proc. R. Soc. London A, 377 (1981), pp. 185-219.

[56] A. Newell, The dynamics of patterns: A survey, in: Propagation in Systems far from Equilib- 
rium (ed. J.E. Wesfeld), Les Houches 1987 (Springer-Verlag, Berlin, Heidelbeg, New York, 1988).

[57] I.M. KRICHEVER, Method of averaging for two-dimensional “integrable" equations, Funct. Anal. Appl., 22 (1988), pp. 200-213;

[58] I.M. KRICHEVER, Spectral theory of two-dimensional periodic operators and its applications, Russian Math. Surveys, 44:2 (1989), pp. 145-225.

[59] I.M. KRICheVer AND D.H. PhONG, On the integrable geometry of soliton equations and $N=$ 2 supersymmetric gauge theories, J. Diff. Geom., 45 (1997), pp. 349-389.

[60] R. Carroll and J.-H. Chang, The Whitham equations revisited, solv-int/9612010.

[61] M.G. Forest AND D.W. MCLAUGHLin, Modulations of sinh-Gordon and sine-Gordon wavetrains, Stud. Appl. Math., 68 (1983), pp. 11-59.

[62] N.M. ERcolani, M.G. Forest and D.W. MCLAughlin, Modulational stability of two-phase sine-Gordon wavetrains, Stud. Appl. Math., 71 (1984), pp. 91-101.

[63] K. TAKASAKI, Dual isomonodromic problems and Whitham equations, Lett. Math. Phys., 43 (1998), pp. 123-135.

[64] K. Окамото, On Fuchs's problem on a torus, I. Funkcial. Ekvac., 14 (1971), pp. 137-152.

[65] - On Fuchs's problem on a torus, II, J. Fac. Sci. Univ. Tokyo, Sect. IA, 24 (1977), pp. 357-371.

[66] —- Déformation d'une équation differéntielle linéaire avec une singularité irrégulière sur un tore, J. Fac. Sci. Univ. Tokyo, Sect. IA, 26 (1979), pp. 501-518.

[67] K. IWASAKI, Moduli and deformation for Fuchsian projective connections on a Riemann surface, J. Fac. Sci. Univ. Tokyo, Sect. IA, 38 (1991), pp. 431-531.

[68] K. IWASAKI, Fuchsian moduli on a Riemann surface - its Poisson structure and PoincaréLefschetz duality, Pacific J. Math., 155 (1992), pp. 319-340.

[69] S. KAWAI, Deformations of complex structures on a torus and monodromy preserving deformations, preprint 1996.

[70] A.M. Levin And M.A. Olshanetsky, Painlevé-Calogero correspondence, alg-geom/9706010;

[71] —- Classical limit of the Knizhnik-Zamolodchikov-Bernard equations as hierarchy of isomonodromic deformations: Free field apoproach, hep-th/9709207. 